Accelerator Department

BROOKHAVEN NATIONAL IABORATORY

Associated Universities, Inc.

Upton, New York

AGS DIVISION TECHNICAI NOTE

№. 71

J.G. Cottingham and G.P. Bagley

December 8, 1969

\title{
FEASIBILITY TEST: FAST BACKIEG BEAM DEFLECTION
}

A test was conducted to determine whether a fast ( $1.0 \mathrm{msec}$ max.) orbit perturbation could be generated using a backleg winding. However, suppose a pulse is applied to a backleg winding thus generating a voltage in that main winding. This voltage would be dropped across the windings of the rest of the magnets in the ring. The resulting counter-acting flux change would completely nullify the flux change in the initial magnet if there were no flux leakages, and if the shunt capacitances and magnet inductances formed a lossless delay Iine. Thus, the object of the test was to determine the transmission characteristics of the magnet ring to a step input to a backleg winding and its departure from an ideal IC line.

A simple test circuit was built which does the following. A large capacitor, Co, is charged through the normally closed contacts of a mercury relay. Approximately once per second the relay is energized connecting the charged capacitor across a 5-turn backleg winding. The specific magnet used for the test was at $E-6$. Photographs of the response across the main windings of E-6, E-5, E-4, E-3 and E-1 were taken using the A-B function of a TEK 454 scope. These voltages were numerically integrated to obtain $2 \int v_{0} d t, \int v_{1} d t$, $\int v_{2} d t, \int v_{3} d t$ and $\int v_{5} d t$ respectively. An extrapolation was made to obtain values of $\int v_{n} d t$ for which the response was not measured. These were combined to form 


$$
2 \int_{0}^{t} v_{0} d t-2 \sum_{n=1}^{18} \cos n \Delta^{\theta} \int_{0}^{t} v_{n} d t
$$

which is proportional to the angular change the beam orbit should experience at the time $t$ for the voltage applied as it passes through this series of magnets. $\Delta \theta$ is $13.15^{\circ} /$ magnet assuming a of 8.75 . This result is plotted in Fig. 1.

The rise of the response at the main winding of E-6 is slowed by the leakage inductance which introduces a time constant of $\left(1-k^{2}\right) \mathrm{L} / \mathrm{R} \approx 21 \mu \mathrm{sec}$ causing $V_{0}$ to be a poor step, where $L$ is the main winding inductance and $R$ is the resistance across the coil packs. However, if $\nabla_{0}$ were a step, an analytic form for the voltage across any coil can be obtained for $t<\frac{\mathrm{L}}{\mathrm{R}}$. The form of the solution involves exp. $\left(n^{2} R C / 4 t\right)$. This was used as the basis for making the extrapolation for those unmeasured responses mentioned above. However, there is no reason to expect that the measured responses should agree closely with this calculated response.

The result of the test indicates that beam perturbations of the order of $100 \mu \mathrm{sec}$ duration can be accomplished using this approach. The net effect of the voltage across the adjacent magnets causes a small negative correction on the beam bending effect of the field change generated in the core of the magnet energized by the backleg winding. The leakage inductance and the resistance across the coil packs effectively put a lower limit on the duration of the beam deflection.

\section{Distr: Department Administration AGS Division Physicists J. Grisoli \\ A. Soukas}




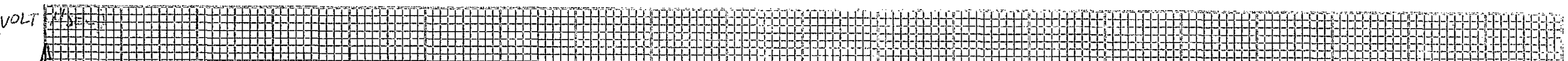

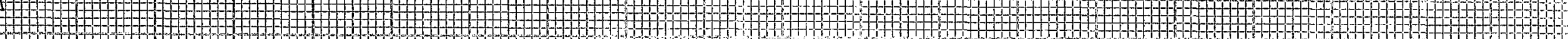

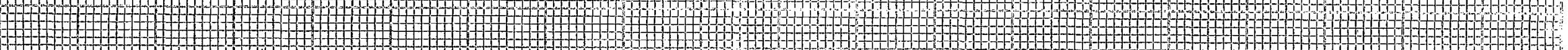

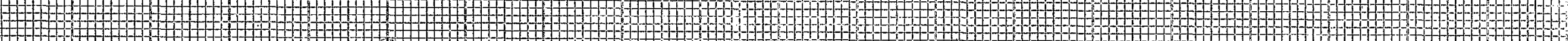
\#

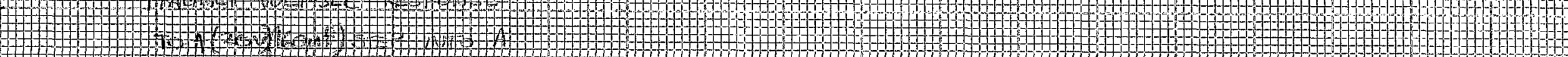
\#+3 Ge+

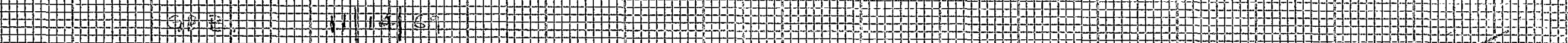
느.

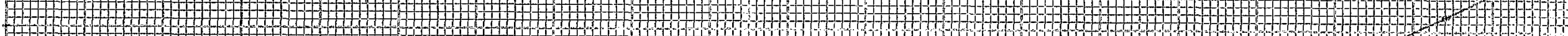

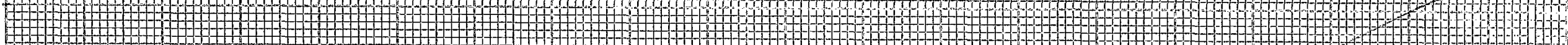

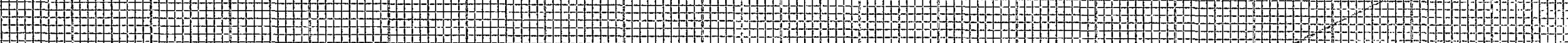
\begin{tabular}{l}
2 \\
\hline
\end{tabular} 17+ + $+2+2$

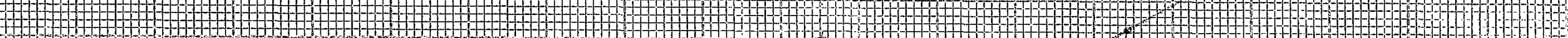
IIt $1+2+2$ 2

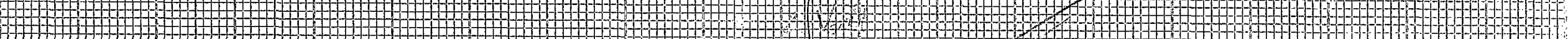
+ 9 Y H+1 $4+\mathrm{Z}+\mathrm{Z}$ -

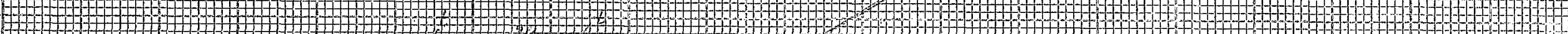

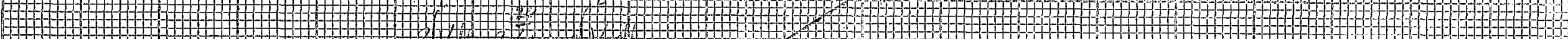
¿ ¿ Z Z + \& I

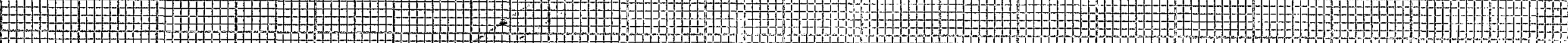
+

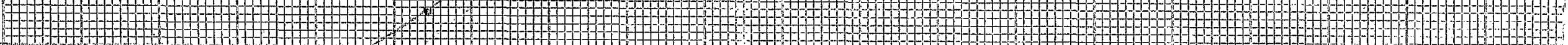

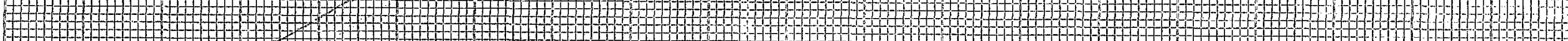
I $6+2=$ L 身 


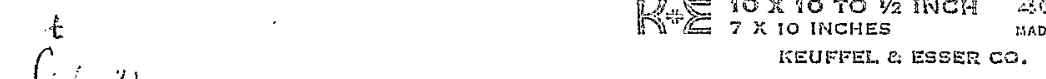

$\int V_{i l} d t$

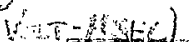

3

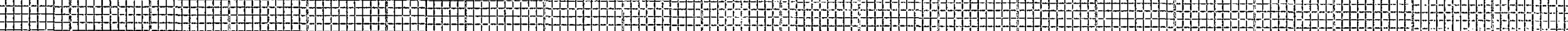

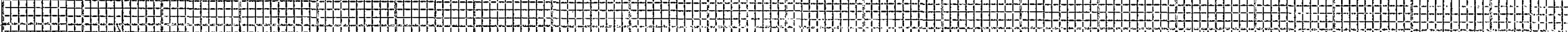

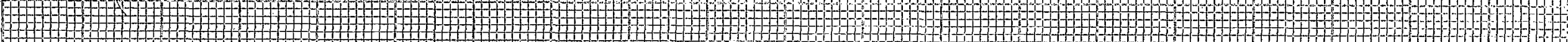

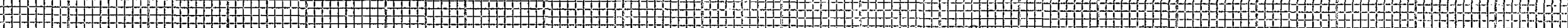

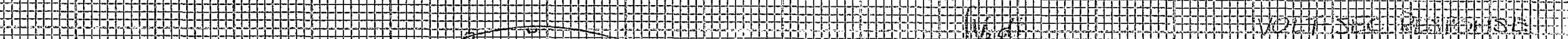

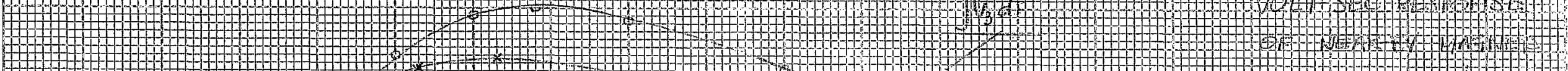

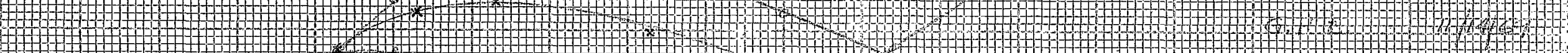

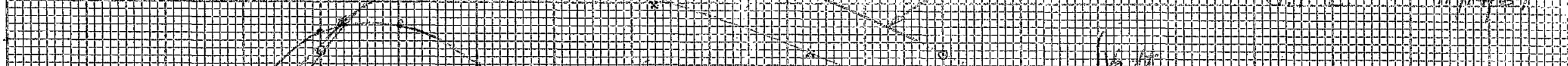

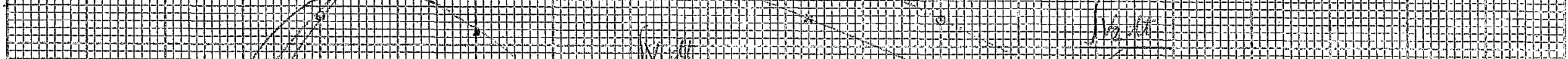

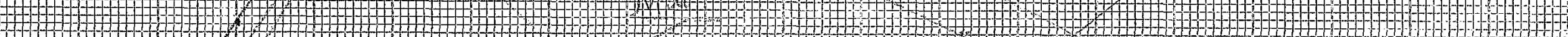

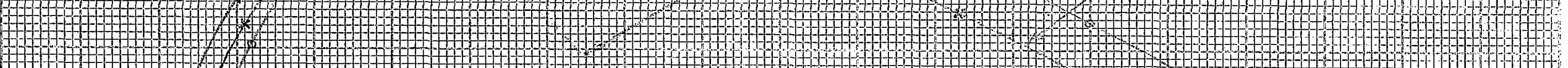

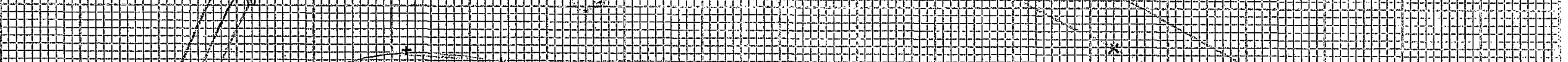

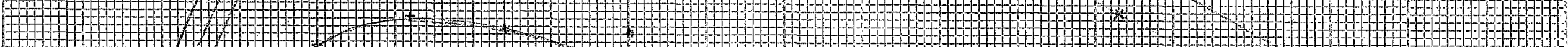

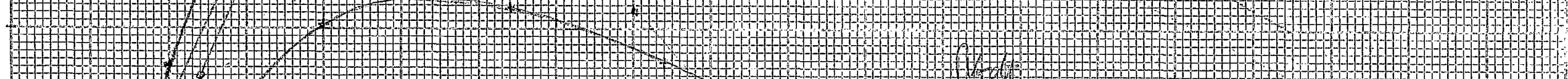

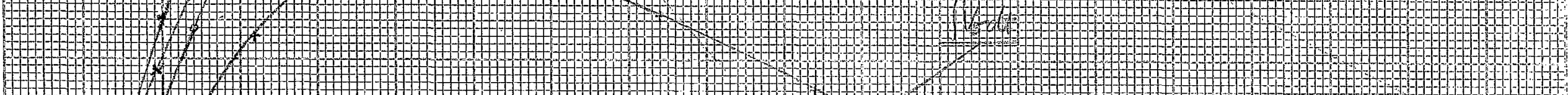

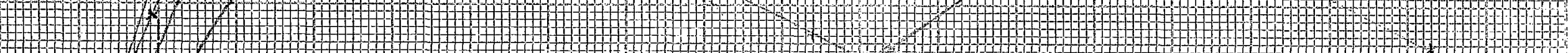

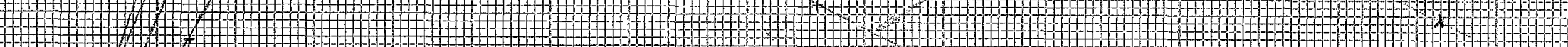

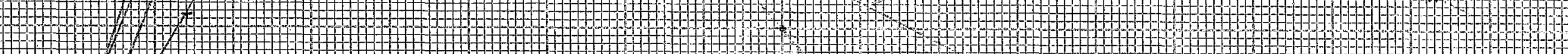

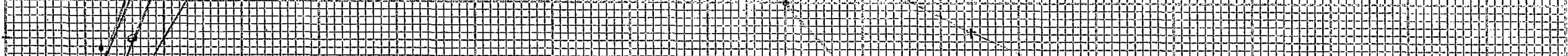

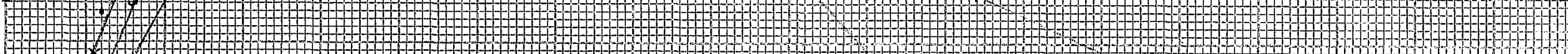

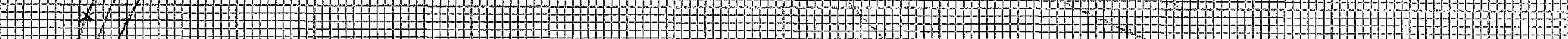

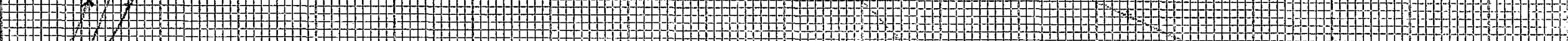

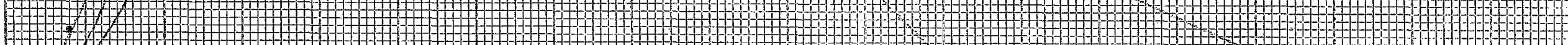

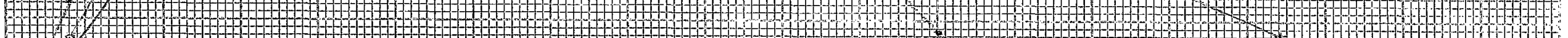

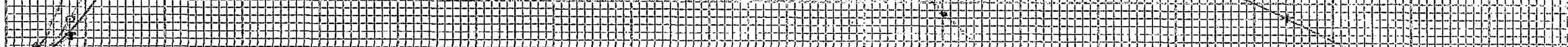

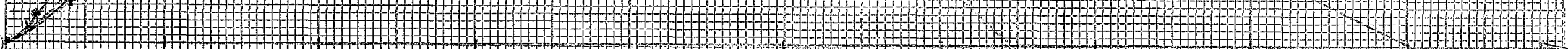

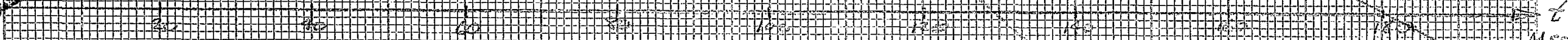

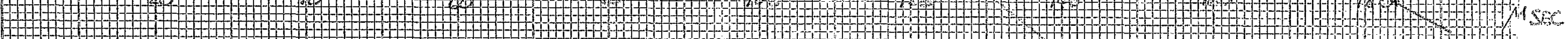

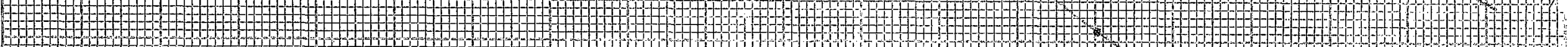

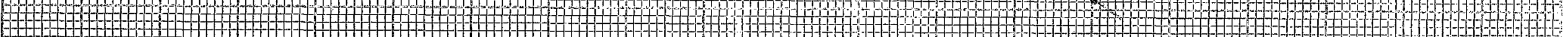

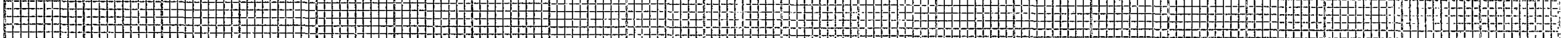

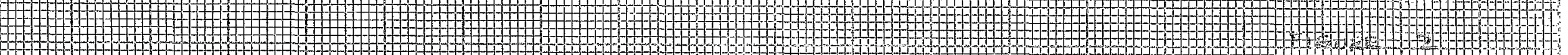

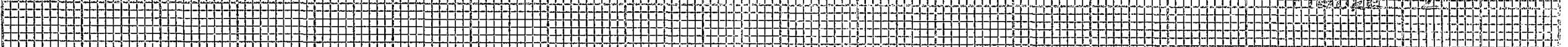

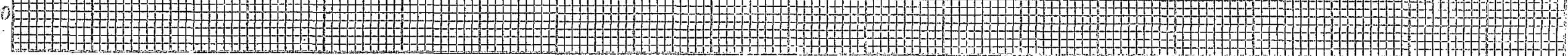




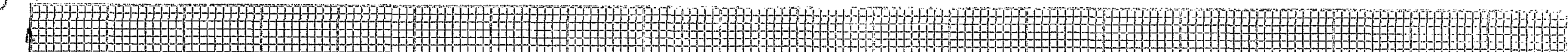

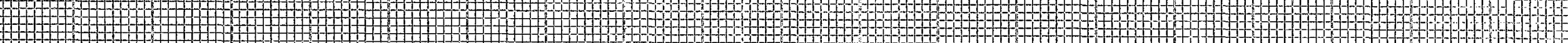

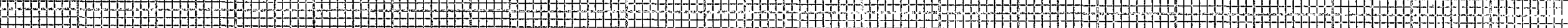

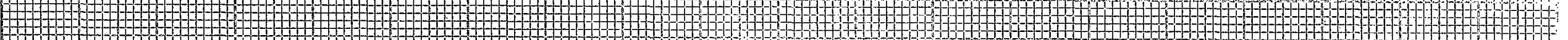

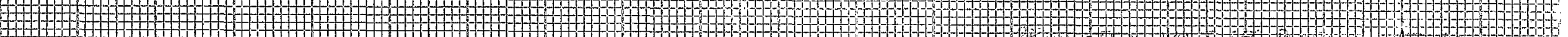

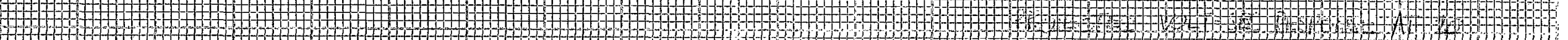

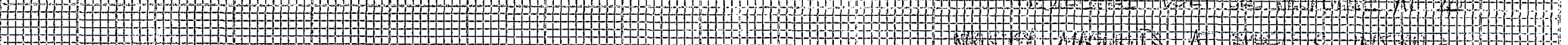

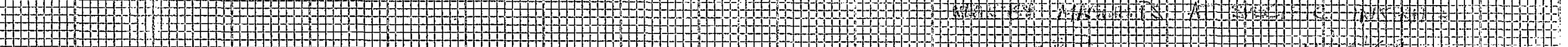

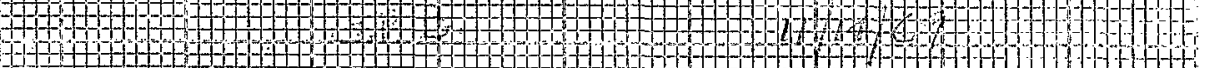

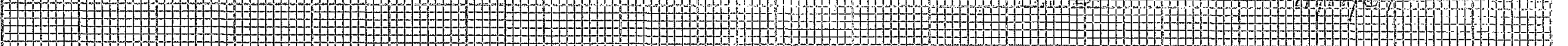

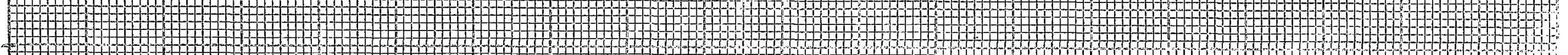

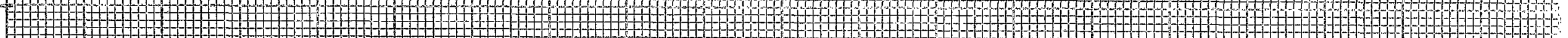

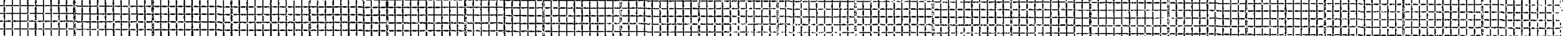

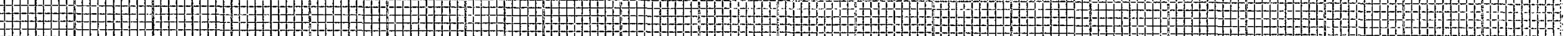

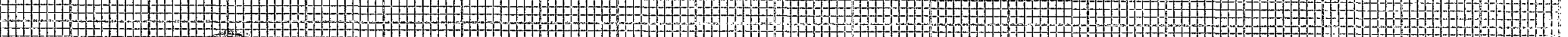

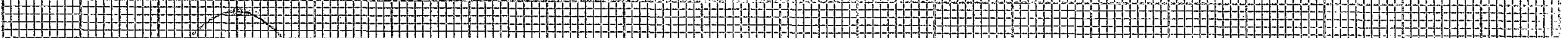

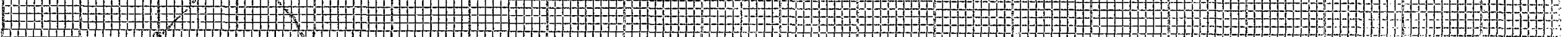

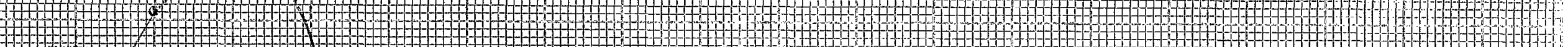

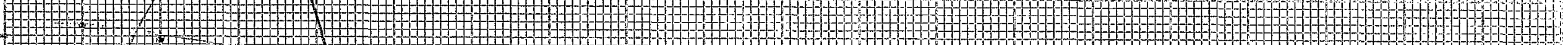

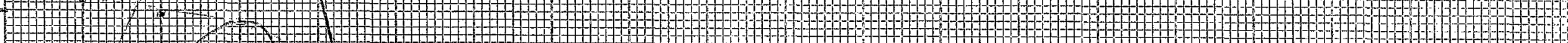

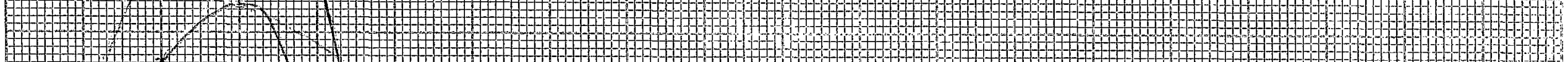

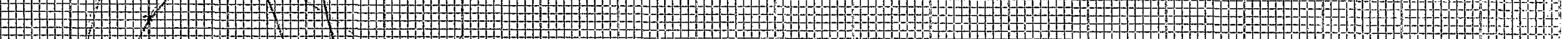

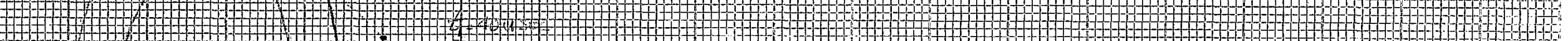

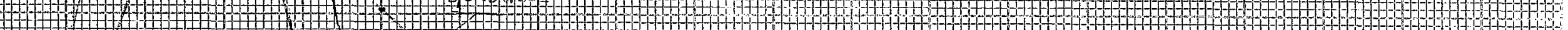

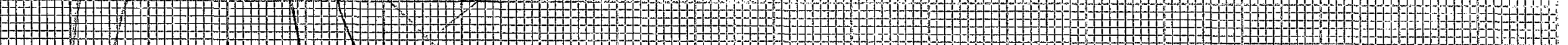

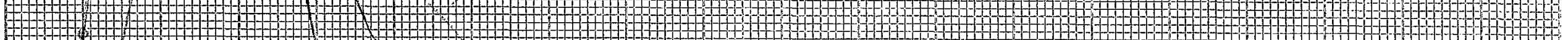

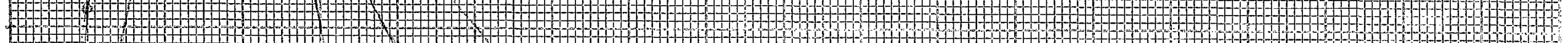

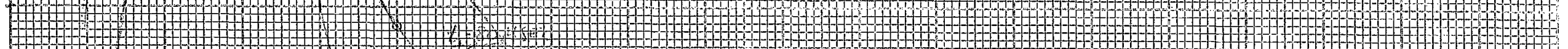

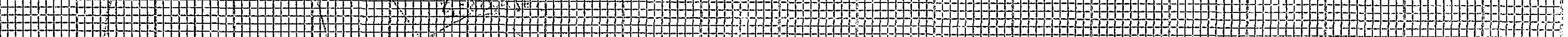

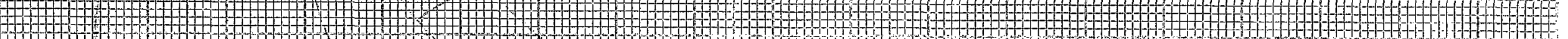

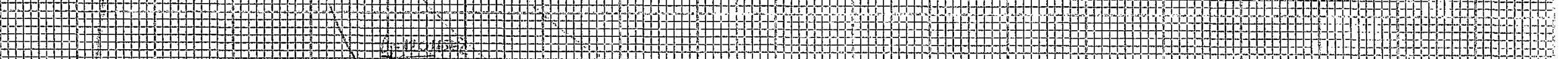

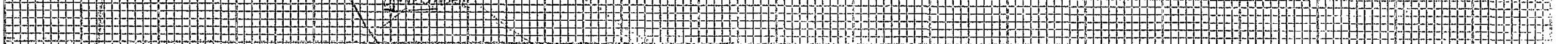

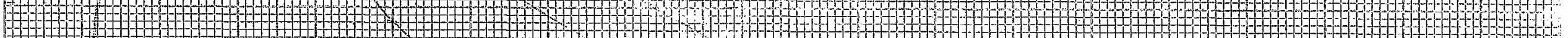

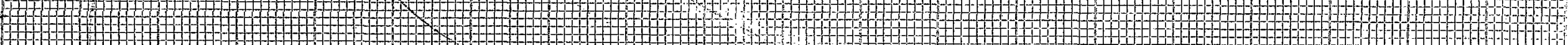

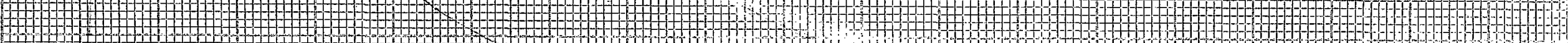

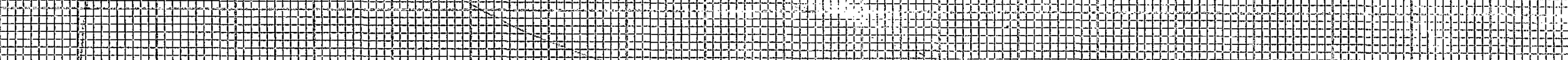

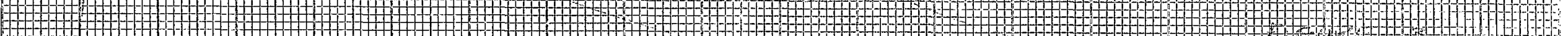

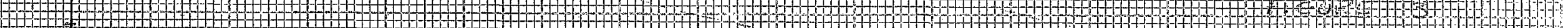

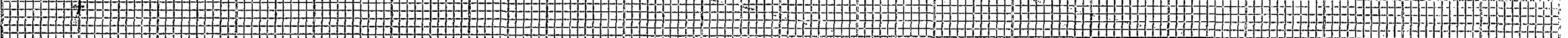

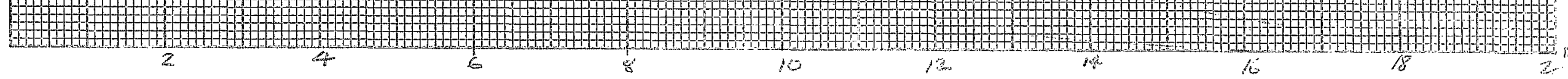


$B Y_{--}$GPB DATE 11/11/69

CHKD. BY DATE

SU UUECT MAGNET VOLTAGE RESPONSE TO $(20 \mathrm{~V})(160 \text { y })^{2}$ BACKIEG WINDING STEP DEPT: DR PROJECT.

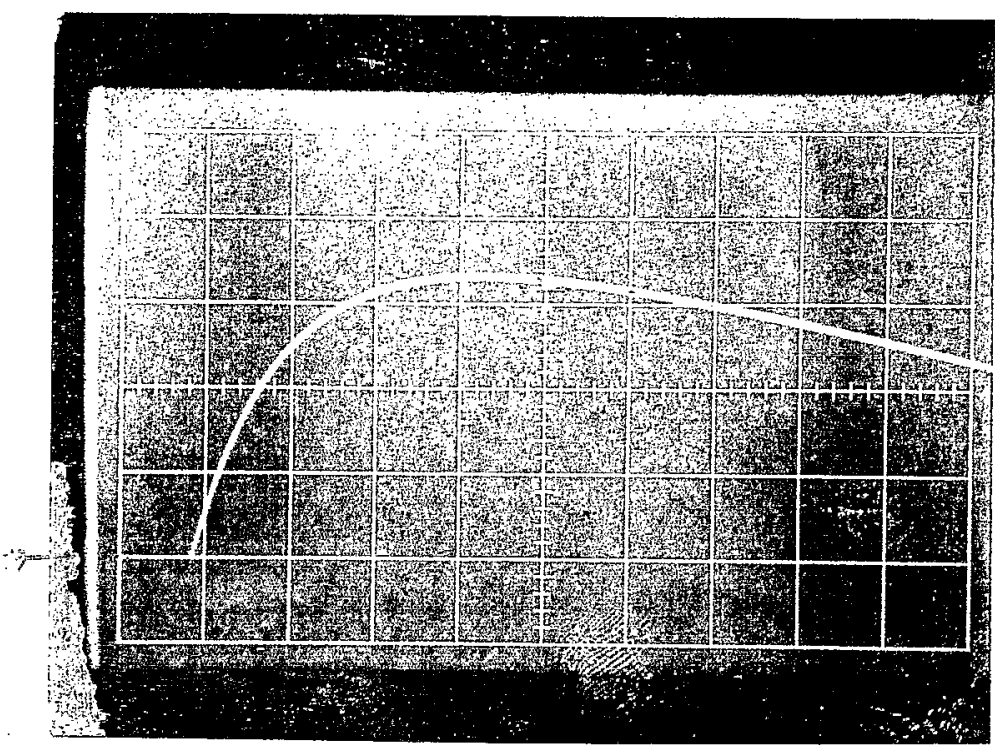

EG., Upper Coil Packs $10 \mathrm{~V} / \mathrm{Div}$, 20 usec/Div

$2 \mathrm{~V}$
SHEET NO. $\square F$

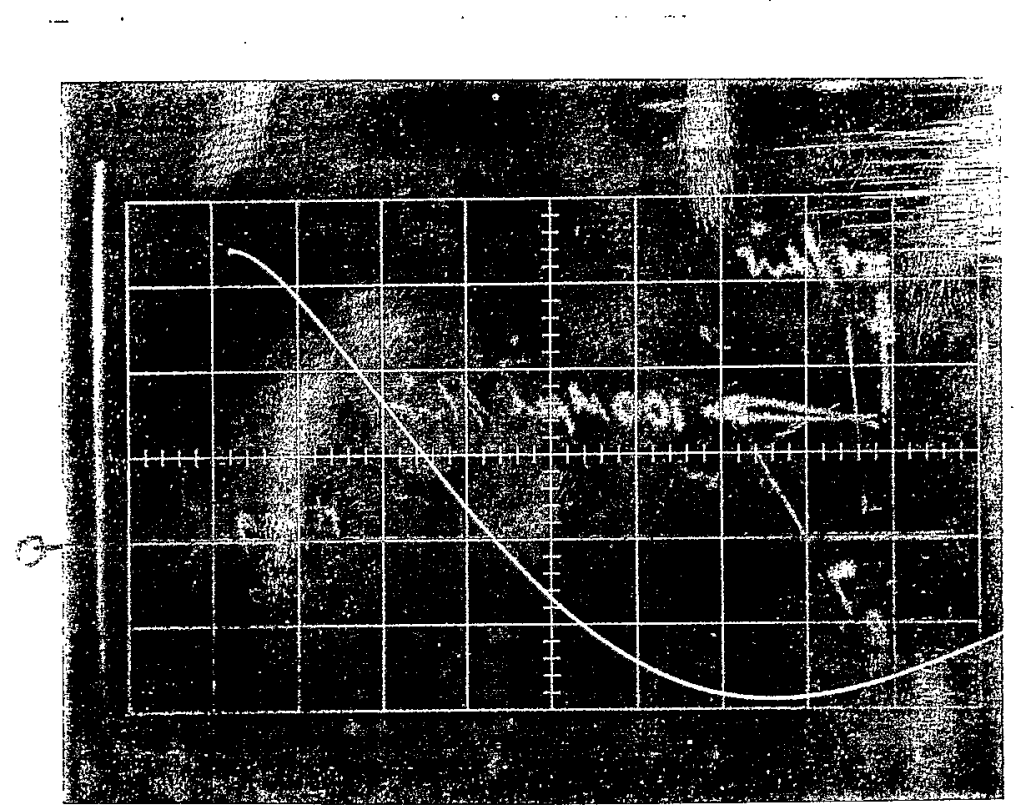

EG., Backleg Winding $5 \mathrm{~V} / \mathrm{Div}, 100$ usec/Div $160 \mu \mathrm{f}$ Charged to $20 \mathrm{~V}$ 
BRDOKHAVEN NATIONAL LABQRATORY

BY GPB DATE.11 1/11]/69

9 פUBUECT.NEAR BY MAGNET_VOITAGE_RESPONSE

CHKD. BY DATE......... TO BACKIEG WINDING STEP

SHEET Na. DF.

DEPT. QR PRDJECT.

$\mathrm{V}_{1}$

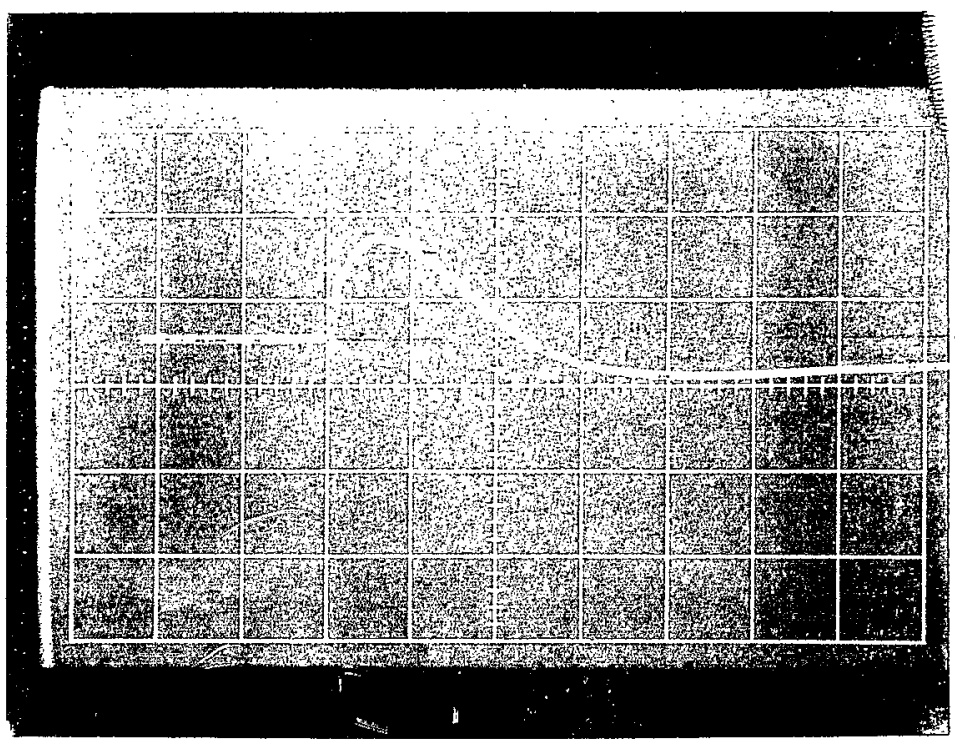

E5, Lower $1.0 \mathrm{~V} / \mathrm{Div}, 20 \mu \mathrm{sec} / \mathrm{Div}$

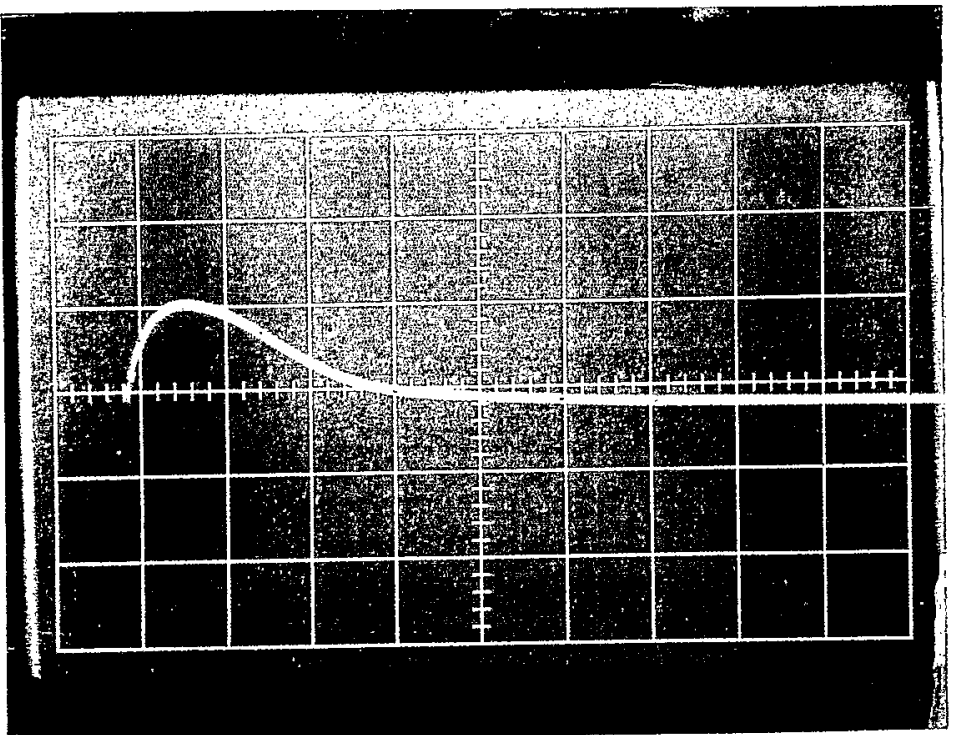

E4, Upper 1.0 V/Div, 20 ,sec/Div 
BRDOKHAVEN NATIONAL LABDRATDRY

BY GPB DATE. $11 / 11 / 69$

CHKD. BY DATE.
SUBJECT. NEAR BY MAGNET VOLTAGE RESPONSE TO BACKIEG WINDING STEP

DEPT. TR PRDJECT.
SHEET ND. $\square F$

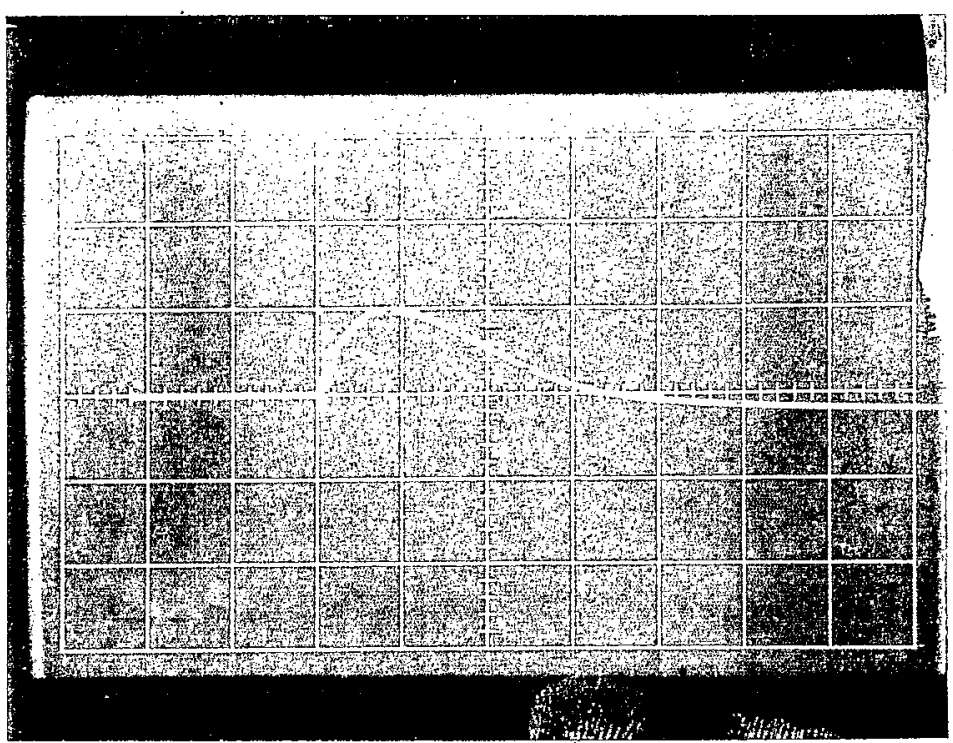

E3, Lower $1.0 \mathrm{~V} / \mathrm{Div}, 20 \mu \mathrm{sec} / \mathrm{Div}$

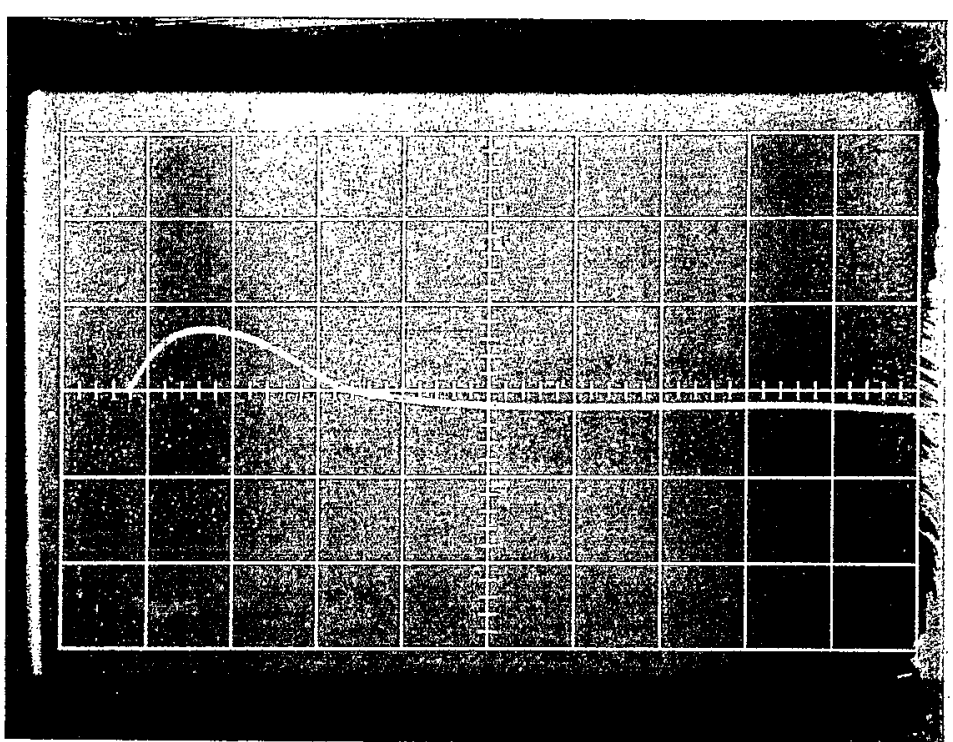

E1, Lower $1.0 \mathrm{~V} / \mathrm{Div}, 20 \mu \mathrm{sec} / \mathrm{Div}$ 